\title{
ON THE POPULATION MODEL WITH A SINE FUNCTION
}

\section{BULA and A.VINTERE}

University of Latvia

Raiņa bulv. 19, Rīga LV 1586, Latvia

E-mail: ibula@lanet.lv; anna.vintere@tl.lv

Received October 4, 2004; revised January 11, 2006

Abstract. In the interval $[0,1]$ function $s_{r}(x)=r \sin \pi x$ behaves similar to logistic function $h_{\mu}(x)=\mu x(1-x)$. We prove that for every $r>\frac{\sqrt{\pi^{2}+1}}{\pi}$ there exists subset $\Lambda \subset[0,1]$ such that $s_{r}: \Lambda \rightarrow \Lambda$ is a chaotic function. Since the logistic function is chaotic in another subset of $[0,1]$ but both functions have similar graphs in $[0,1]$ we conclude that it can lead to errors in practice.

Key words: logistic function, sine function, chaotic function

\section{Introduction}

Difference equations usually describe the evolution of certain phenomena over the course of time. If a certain population has discrete generation, the size of the $(n+1)$-th generation $x(n+1)$ is a function of the $n$th generation $x(n)$. This relation expresses itself in the difference equation

$$
x(n+1)=f(x(n)) .
$$

We start in the point $x_{0}$ and use the following notation

$$
f^{2}\left(x_{0}\right)=f\left(f\left(x_{0}\right)\right), \quad f^{3}\left(x_{0}\right)=f\left(f\left(f\left(x_{0}\right)\right)\right) .
$$

Letting $x(n)=f^{n}\left(x_{0}\right)$, we have

$$
x(n+1)=f^{n+1}\left(x_{0}\right)=f\left(f^{n}\left(x_{0}\right)\right)=f(x(n)) .
$$

The exponential models have only a limited predictive power in population problems since as time passes the predicted population becomes so large that it is no longer realistic. For most biological species it is valid that the population increases until it reaches a certain upper limit. Then, due to the limitations of 
available resources, the creatures will become testy and engage in competition for those limited resources. In 1845 Pierre-Francois Verhulst had offered for investigation of population the following mathematical model

$$
x(n+1)=\mu x(n)(1-x(n)),
$$

where $x(n)$ is the size of a population at time $n$ and $\mu$ is the rate of growth of the population. This equation is the simplest nonlinear first-order difference equation but it describes a complicated dynamics. The quadratic function $h \mu(x)=\mu x(1-x), x \in[0,1]$, is called also logistic function. The logistic function is widely studied (see, for example, $[3,4,5,6]$ ).

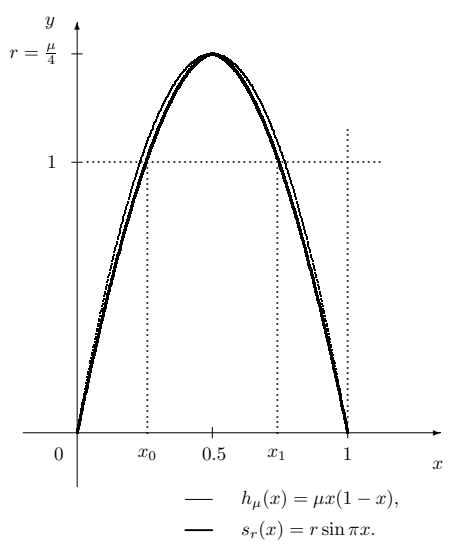

Figure 1. The logistic and sin functions.

The function

$$
s_{r}(x)=r \sin \pi x
$$

also has a similar behaviour in the interval $[0,1]$, here $r>0$ is a parameter (see Fig. 1). If we fix $x_{0}$ and consider the orbit

$$
\left\{x_{0}, s_{r}\left(x_{0}\right), s_{r}\left(s_{r}\left(x_{0}\right)\right), \ldots,\right\}
$$

then similar to logistic function the dynamics of $s_{r}(x)$ is very complicated. There exist more functions with similar behaviour in $[0,1]$, but we consider sine function, because it is very popular to approximate real functions with trigonometric series, especially by using Fourier series. Let $D \subset \mathbf{R}$.

Definition 1. (R.Devaney, [2]) The function $f: D \rightarrow D$ is chaotic if

a) the periodic points of $f$ are dense in $D$,

b) $f$ is topologically transitive in $D$,

c) $f$ exhibits sensitive dependence on initial conditions in $D$. 
We prove that for every $r>\frac{\sqrt{\pi^{2}+1}}{\pi}$ there exists a subset $\Lambda \subset[0,1]$ such that $s_{r}: \Lambda \rightarrow \Lambda$ is chaotic function by Devaney's definition. The chaotic function preserves a certain amount of regularity and mixes the domain well. Even very small changes in the initial position may result in dramatically different results in values of the iterated function.

\section{The Dynamics of Sine Function}

In this section we prove that for every $r>\frac{\sqrt{\pi^{2}+1}}{\pi}$ there exists a subset $\Lambda \in[0,1]$ such that $s_{r}: \Lambda \rightarrow \Lambda$ is a chaotic function. At first, we clear up conceptions of the Devaney definition of chaotic functions (see [3]). Let $D \subset \mathbf{R}$.

Definition 2. Let $A$ be a subset of $B \subset \mathbf{R}$. Then $A$ is dense in $B$ if for each point $x \in B$ and each $\varepsilon>0$ there exists $y \in A$ such that $|x-y|<\varepsilon$.

Definition 3. The function $f: D \rightarrow D$ is topologically transitive on $D$ if for any two points $x$ and $y$ in $D$ and any $\varepsilon>0$, there exists $z$ in $D$ such that $|z-x|<\varepsilon$ and $\left|f^{n}(z)-y\right|<\varepsilon$ for some $n$.

Definition 4. The function $f: D \rightarrow D$ exhibits sensitive dependence on initial conditions if there exists a $\delta$ such that for any $x$ in $D$ and any $\varepsilon>$ 0 , there is a $y$ in $D$ and a natural number $n$ such that $|x-y|<\varepsilon$ and $\left|f^{n}(x)-f^{n}(y)\right|>\delta$.

The result by Banks, Brooks, Cairns, Davis and Stacey [1] demonstrates that when the domain of definition of a continuous function is infinite, then the density of periodic points and topological transitivity imply sensitive dependence on initial conditions.

Theorem 1. ([1]) Let $D$ be an infinite subset of the real numbers and $f$ : $D \rightarrow D$ be continuous. If $f$ is topologically transitive on $D$ and the periodic points of $f$ are dense in $D$, then $f$ is chaotic on $D$.

In our case $s_{r}(x)=r \sin \pi x$ is continuous function in segment $[0,1]$. If the function $f$ is differentiable at each point of $D \subset \mathbf{R}$ and both $f$ and $f^{\prime}$ are continuous then $f$ is said to be continuously differentiable or to be a $C^{1}$ function. For this class of functions the following result is valid.

Theorem 2. ([5], p.38-39, Theorem 5.2 and 5.3) Let $f: \mathbf{R} \rightarrow \mathbf{R}$ be a $C^{1}$ function and $I_{1}, \ldots, I_{p}$ be $p$ disjoint closed bounded intervals with $p \geq 2$. Let $J=\cup_{j=1}^{p} I_{j}$. Assume that $f\left(I_{j}\right) \supset J$ for $1 \leq j \leq p$. Also assume that there is a constant $\lambda>1$ such that $\left|f^{\prime}(x)\right| \geq \lambda$ for $x \in J \cap f^{-1}(J)$. Let $\Lambda=\cap_{k=0}^{\infty} f^{-k}(J)$. Then the following statements are valid: a) $\Lambda$ is a Cantor set, b) the set of periodic points of $f$ is dense in $\Lambda, c) f$ is topologically transitive on $\Lambda$.

We consider the set $\Lambda_{1}=\left[0, \frac{1}{\pi} \arcsin \frac{1}{r}\right] \cup\left[1-\frac{1}{\pi} \arcsin \frac{1}{r}, 1\right]\left(\right.$ or $\Lambda_{1}=$ $\left\{x \mid s_{r}(x)\right.$ is in $\left.\left.[0,1]\right\}, r>1\right)$. Now we prove the following proposition. 
Proposition 1. If $s_{r}(x)=r \sin \pi x$ and $r>\frac{\sqrt{\pi^{2}+1}}{\pi} \approx 1.05$, then

$$
\inf \left\{\left|s_{r}^{\prime}(x)\right| \mid x \in \Lambda_{1}\right\}>1 .
$$

Proof. The derivative of $s_{r}$ is given by $s_{r}^{\prime}(x)=r \pi \cos \pi x$. Also the estimate $s_{r}^{\prime \prime}(x)=-r \pi^{2} \sin \pi x<0$ is valid for all $\left.x \in\right] 0,1[$. So the smallest value of $\left|s_{r}^{\prime}(x)\right|$ on $\Lambda_{1}$ occurs where $s_{r}(x)=1$. Solving equation

$$
1=s_{r}(x)=r \sin \pi x
$$

we get $x_{0}=\frac{1}{\pi} \arcsin \frac{1}{r}$ and $x_{1}=1-\frac{1}{\pi} \arcsin \frac{1}{r}$ (see Fig.1). For these points we have

$$
\begin{aligned}
& s_{r}^{\prime}\left(x_{0}\right)=r \pi \cos \left(\pi \cdot \frac{1}{\pi} \arcsin \frac{1}{r}\right)=\pi \sqrt{r^{2}-1} \\
& s_{r}^{\prime}\left(x_{1}\right)=r \pi \cos \left(\pi \cdot\left(1-\frac{1}{\pi} \arcsin \frac{1}{r}\right)\right)=-\pi \sqrt{r^{2}-1} .
\end{aligned}
$$

We need to satisfy the inequality $\left|s_{r}^{\prime}\left(x_{0,1}\right)\right|=\pi \sqrt{r^{2}-1}>1$. From it we find $r>\frac{\sqrt{\pi^{2}+1}}{\pi}$. Therefore if $r>\frac{\sqrt{\pi^{2}+1}}{\pi}$ then

$$
\inf \left\{\left|s_{r}^{\prime}(x)\right| \mid x \in \Lambda_{1}\right\}>1 \text {. }
$$

Theorem 3. If $r>\frac{\sqrt{\pi^{2}+1}}{\pi}>1$, then there exist a subset $\Lambda \subset[0,1]$ such that $s_{r}: \Lambda \rightarrow \Lambda$ is a chaotic function.

Proof. The function $s_{r}(x)=r \sin \pi x$ is a $C^{1}$ function. Since

$$
x_{0}=\frac{1}{\pi} \arcsin \frac{1}{r}<\frac{1}{2}<x_{1}=1-\frac{1}{\pi} \arcsin \frac{1}{r}
$$

(see Fig.1), then

$$
I_{1}=\left[0, \frac{1}{\pi} \arcsin \frac{1}{r}\right] \text { and } I_{2}=\left[1-\frac{1}{\pi} \arcsin \frac{1}{r}, 1\right]
$$

are two disjoint closed bounded intervals. Besides $s_{r}\left(I_{j}\right) \supset I_{1} \cup I_{2}, j=1,2$. By Proposition 1 there is a $\lambda_{r}=\inf \left\{\left|s_{r}^{\prime}(x)\right| \mid x \in \Lambda_{1}\right\}>1$ such that for $x \in\left(I_{1} \cup I_{2}\right) \cap s_{r}^{-1}\left(I_{1} \cup I_{2}\right)$ we have $\left|s_{r}^{\prime}(x)\right| \geq \lambda_{r}$. Then by Theorem 2

$$
\Lambda=\cap_{k=0}^{\infty} s_{r}^{-k}\left(I_{1} \cup I_{2}\right)
$$

is a Cantor set, that is, it is an infinite subset of the real numbers, and $s_{r}$ : $\Lambda \rightarrow \Lambda$ is continuous. By Theorem 2 the set of periodic points of $s_{r}$ are dense in $\Lambda$ and $s_{r}$ is topologically transitive on $\Lambda$. From Theorem 1 it follows that $s_{r}$ is chaotic on $\Lambda$. 


\section{Comparison of Sine Function with Logistic Function}

We make three remarks.

1. Suppose we have 100 points of graph of function $s_{r}(x)=r \sin \pi x, r>$ $\frac{\sqrt{\pi^{2}+1}}{\pi}$, and we do not know that exactly this function is considered. What is a possibility that if we make approximation we choose $s_{r}$ ? Graphics of $s_{r}$ and $h_{\mu}$ are similar (see Fig. 1 in case $r=\frac{\mu}{4}$ ). Since quadratic function is more simple, therefore it is high possibility that we make approximation with quadratic map. If this quadratic map is $h_{\mu}=\mu x(1-x), \mu>4$, then we know that there exists a subset $\Lambda_{h} \subset[0,1]$ such that $h_{\mu}: \Lambda_{h} \rightarrow \Lambda_{h}$ is chaotic $([3,5])$. Also there exists a subset $\Lambda_{s} \subset[0,1]$ such that $s_{r}: \Lambda_{s} \rightarrow$ $\Lambda_{s}$ is chaotic. But $\Lambda_{h} \neq \Lambda_{s}$ (for example, if $r=\frac{\mu}{4}$ then

$$
x_{0}=\frac{1}{\pi} \arcsin \frac{1}{r}, \quad x_{1}=1-\frac{1}{\pi} \arcsin \frac{1}{r}
$$

belong to $\Lambda_{s}$ but not to $\left.\Lambda_{h}\right)$.

2. The function $s_{r}(x)=r \sin \pi x, x \in[0,1]$, has the following properties:

(1) it is smooth function and $s_{r}:[0,1] \rightarrow \mathbf{R}$,

(2) it has maximum point $x_{m}=\frac{1}{2}$ and $s_{r}^{\prime \prime}\left(\frac{1}{2}\right)=-r \pi^{2} \neq 0$,

(3) it is monotone in segments $\left[0, x_{m}[\right.$ and $\left.] x_{m}, 1\right]$,

(4) it has negative the Schwarz derivative, i.e., $\forall x \in[0,1] \backslash\left\{x_{m}\right\}$

$$
S_{s_{r}}(x)=\frac{s_{r}^{\prime \prime \prime}(x)}{s_{r}^{\prime}(x)}-\frac{3}{2}\left(\frac{s_{r}^{\prime \prime}(x)}{s_{r}^{\prime}(x)}\right)^{2}=-\pi^{2}\left(1+\frac{3}{2}\left(\frac{\sin \pi x}{\cos \pi x}\right)^{2}\right)<0 .
$$

By [4] in this case there exists bifurcation diagram for $s_{r}$ and it is similar to $h_{\mu}$.

3. Let $h_{\mu}(x)=\mu x(1-x), \mu>4$, and let $\Lambda_{h} \subset[0,1]$ is a set in which $h_{\mu}$ is chaotic. It is known that if $x \notin \Lambda_{h}$ then the limit of $h_{\mu}^{n}(x)$ is equal to minus infinity as $n$ goes to infinity $([3,5,6])$. Let $s_{r}(x)=r \sin \pi x, r>\frac{\sqrt{\pi^{2}+1}}{\pi}$, and let $\Lambda_{s} \subset[0,1]$ is a set in which $h_{r}$ is chaotic. Since $|r \sin \pi x| \leq 1$ then if $x \notin \Lambda_{s}$ then the limit of $s_{r}^{n}(x)$ is not infinity as $n$ goes to infinity. This is a very interesting difference.

For example, we consider point $x_{m}=\frac{1}{2}$. If $r \in \mathbf{N}$ then

$$
s_{r}\left(\frac{1}{2}\right)=r>1, \quad s_{r}^{2}\left(\frac{1}{2}\right)=r \sin \pi r=0, \quad s_{r}^{k}\left(\frac{1}{2}\right)=0, \quad k \geq 2,
$$

i.e., $x_{m}$ is eventually fixed point and $x_{m}$ returns to the interval $[0,1]$ under iteration of $s_{r}$. If $r=\frac{m}{2}, m \in \mathbf{N}$ and $m$ is an odd number then

$s_{r}\left(\frac{1}{2}\right)=\frac{m}{2}, s_{r}^{2}\left(\frac{1}{2}\right)=\frac{m}{2} \sin \frac{\pi m}{2}=\left\{\begin{array}{l}\frac{m}{2}, m=1+4 l, l \in \mathbf{N}, \\ -\frac{m}{2}, m=3+4 l, l \in \mathbf{N},\end{array} \quad s_{r}^{3}\left(\frac{1}{2}\right)=\frac{m}{2}\right.$. 
We see that if $r=\frac{1+4 l}{2}, l \in \mathbf{N}$, then $x_{m}=\frac{1}{2}$ is eventually a fixed point but it does not belong to [0,1]. If $r=\frac{3+4 l}{2}, l \in \mathbf{N}$, then $x_{m}=\frac{1}{2}$ is eventually a periodic point with period 2 and it also leaves the interval $[0,1]$ under iteration of $s_{r}$.

Generally, if $r>2$ then there exist two points $x_{1}, x_{2} \in[0,1]$ such that

$$
s_{r}\left(x_{1}\right)=s_{r}\left(x_{2}\right)=2, \quad s_{r}^{2}\left(x_{1}\right)=s_{r}^{2}\left(x_{2}\right)=r \sin r \pi=0,
$$

i.e., $x_{1}$ and $x_{2}$ are eventually fixed points and they return back to the interval $[0,1]$. We also remark that if $r>2$ then for every $y \in] 1,2]$ there exist $x_{3}$ and $x_{4}$ in $[0,1]$ such that

$$
s_{r}\left(x_{3}\right)=s_{r}\left(x_{4}\right)=y, \quad s_{r}^{2}\left(x_{3}\right)=s_{r}^{2}\left(x_{4}\right) \in[-r, 0] .
$$

Since $s_{r}([-1,0])=[-r, 0]$ and $s_{r}([-2,-1])=[0, r]$ then there exist points such that they leave the interval $[0,1]$ in the first iteration and return back to the interval $[0,1]$ under iteration of $s_{r}$.

\section{Conclusion}

We conclude that functions with similar graphs have different "chaotic" properties. If we make approximation of real data with one chaotic function but in reality this data was generated by another chaotic function then it is possible that the errors of our forecast are higher than we expected. In the case of chaotic functions the long-term forecast is not possible and we can not define precisely exact functions thus the choice of models with chaotic functions is unpredictable.

\section{References}

[1] J. Banks, J. Brooks, G. Cairns, G. Davis and P. Stacey. On Devaney's definition of chaos. Amer. Math. Monthly, 99, 332 - 334, 1992.

[2] R. Devaney. An Introduction to Chaotic Dynamical Systems, 2nd edition. Addison-Wesley, 1989.

[3] R. Holmgren. A First Course in Discrete Dynamical Systems. Springer-Verlag, 1996.

[4] H.O. Peitgen, H. Jürgens and D. Saupe. CHAOS - Bausteine der Ordnung. Klett-Cotta/Springer-Verlag, 1994.

[5] C. Robinson. Dynamical Systems. Stability, Symbolic Dynamics, and Chaos. CRC Press, 1995.

[6] H. Zeitler and W. Neidhardt. Fraktale und Chaos. Eine Einfürung. Wissenschaftliche Buchgesellschaft, Darmstadt, 1994. 